2016-02-09

\title{
Ecological and methodological drivers of species' distribution and phenology responses to climate change
}

\section{Brown, CJ}

http://hdl.handle.net/10026.1/10109

\subsection{1/gcb.13184 \\ GLOBAL CHANGE BIOLOGY}

All content in PEARL is protected by copyright law. Author manuscripts are made available in accordance with publisher policies. Please cite only the published version using the details provided on the item record or document. In the absence of an open licence (e.g. Creative Commons), permissions for further reuse of content should be sought from the publisher or author. 


\title{
Ecological and methodological drivers of species' distribution and phenology responses to climate change
}

\author{
CHRISTOPHER J. BROWN ${ }^{1}$, MARY I. O'CONNOR ${ }^{2}$, ELVIRA S. POLOCZANSKA ${ }^{1,3}$, \\ DAVID S. SCHOEMAN ${ }^{4}$, LAUREN B. BUCKLEY ${ }^{5}$, MICHAEL T. BURROWS ${ }^{6}$, \\ CARLOS M. DUARTE7 , BENJAMIN S. HALPERN $8,9,10$, JOHN M. PANDOLFI $^{11}$, \\ CAMILLE PARMESAN ${ }^{12,13}$ and ANTHONY J. RICHARDSON ${ }^{3,14}$ \\ ${ }^{1}$ The Global Change Institute, The University of Queensland, St Lucia, Qld 4072, Australia, ${ }^{2}$ Department of Zoology and \\ Biodiversity Research Centre, University of British Columbia, Vancouver, BC, Canada V6T1Z4, ${ }^{3}$ CSIRO Oceans and Atmosphere, \\ EcoSciences Precinct, Dutton Park, Brisbane, Qld 4102, Australia, ${ }^{4}$ School of Science and Engineering, University of Sunshine \\ Coast, Maroochydore, Qld 4558, Australia, ${ }^{5}$ Department of Biology, University of Washington, Seattle, WA 98115-1800, USA, \\ ${ }^{6}$ Department of Ecology, Marine Institute, Scottish Association for Marine Science, Oban, Argyll PA37 1QA, UK, ${ }^{7}$ Red Sea \\ Research Center (RSRC), King Abdullah University of Science and Technology (KAUST), Thuwal 23955-6900, Saudi Arabia, \\ ${ }^{8}$ National Center for Ecological Analysis and Synthesis, 735 State St. Suite 300, Santa Barbara, CA 93101, USA, ${ }^{9}$ Bren School of \\ Environmental Science and Management, University of California, Santa Barbara, CA 93106, USA, ${ }^{10}$ Imperial College London, \\ Silwood Park Campus, Buckhurst Road, Ascot SL57PY, UK, ${ }^{11}$ School of Biological Sciences, ARC Centre of Excellence for Coral \\ Reef Studies, The University of Queensland, St Lucia, Qld 4072, Australia, ${ }^{12}$ Marine Institute, Plymouth University, Drakes \\ Circus, Plymouth, Devon PL4 8AA, UK, ${ }^{13}$ Department of Geological Sciences, University of Texas at Austin, Austin, TX 78712, \\ USA, ${ }^{14}$ School of Mathematics and Physics, Centre for Applications in Natural Resource Mathematics, The University of \\ Queensland, St Lucia, Qld 4072, Australia
}

\begin{abstract}
Climate change is shifting species' distribution and phenology. Ecological traits, such as mobility or reproductive mode, explain variation in observed rates of shift for some taxa. However, estimates of relationships between traits and climate responses could be influenced by how responses are measured. We compiled a global data set of 651 published marine species' responses to climate change, from 47 papers on distribution shifts and 32 papers on phenology change. We assessed the relative importance of two classes of predictors of the rate of change, ecological traits of the responding taxa and methodological approaches for quantifying biological responses. Methodological differences explained $22 \%$ of the variation in range shifts, more than the $7.8 \%$ of the variation explained by ecological traits. For phenology change, methodological approaches accounted for $4 \%$ of the variation in measurements, whereas $8 \%$ of the variation was explained by ecological traits. Our ability to predict responses from traits was hindered by poor representation of species from the tropics, where temperature isotherms are moving most rapidly. Thus, the mean rate of distribution change may be underestimated by this and other global syntheses. Our analyses indicate that methodological approaches should be explicitly considered when designing, analysing and comparing results among studies. To improve climate impact studies, we recommend that (1) reanalyses of existing time series state how the existing data sets may limit the inferences about possible climate responses; (2) qualitative comparisons of species' responses across different studies be limited to studies with similar methodological approaches; (3) meta-analyses of climate responses include methodological attributes as covariates; and (4) that new time series be designed to include the detection of early warnings of change or ecologically relevant change. Greater consideration of methodological attributes will improve the accuracy of analyses that seek to quantify the role of climate change in species' distribution and phenology changes.
\end{abstract}

Keywords: fishing, global warming, marine ecosystem, meta-analysis, publication bias, range edge, range shift, season, time series, tropics

Received 25 June 2015; revised version received 11 October 2015 and accepted 13 November 2015

Correspondence: Present address: Christopher J. Brown, Australian Rivers Institute, Griffith University, 170 Kessels Road, Nathan, Qld, Australia, tel. +61 737359 268, fax +61 737355 044, e-mail: christopher.brown@griffith.edu.au

\section{Introduction}

A large number of marine (Poloczanska et al., 2013) and terrestrial (Parmesan \& Yohe, 2003) species have shifted their distributions and phenology in recent decades, indicating that climate change is driving a global 
biological response. For example, recent climate change has: driven the invasion of pest species (Ling et al., 2008), contributed to declines in commercially important fish species (Beaugrand \& Kirby, 2010) and appears to be increasing mismatch in the seasonal timing between predators and their prey (Edwards \& Richardson, 2004; Barbraud \& Weimerskirch, 2006).

Despite an overall broad consistency in species' responses to climate change, there is considerable variability in magnitudes and patterns of responses (Parmesan, 2007; Poloczanska et al., 2013; Sunday et al., 2015). Variability poses a challenge to ecological science and management of species impacted by climate change, because it hinders predictions of future responses. Analyses across many species have examined how combinations of taxonomic identity, ecological traits and local environmental variables may explain variability in responses (Perry et al., 2005; Wolkovich et al., 2012; Pinsky et al., 2013; Poloczanska et al., 2013; Sunday et al., 2015). All of these approaches base their inferences on standardized measures of distribution and phenology, yet observed responses to climate change may also depend on how distribution and phenology are measured (Wolkovich et al., 2012).

Measurements of distribution and phenology are influenced by a suite of decisions that are made in two stages of all studies: their sampling design and data analysis (Brown et al., 2011; Bates et al., 2014a). In the sampling design phase, researchers decide how species' variables are measured. For example, distribution can be measured as mean latitude of a populations' geographical extent (e.g. Perry et al., 2005) or by measuring the most extreme latitudes where a species is found (e.g. Robinson et al., 2015). Similarly, the phenology of breeding events can be measured by censusing a population throughout a season to determine the peak breeding date, or as the first and last individuals to breed (e.g. Fitter \& Fitter, 2002; Barbraud \& Weimerskirch, 2006). Measures of distribution and phenology based on the most extreme individuals rather than variables that represent the distribution of individuals within a population may lead to very different estimates of climate change response rates. For example, single individuals may by chance have extreme responses (Brown et al., 2011), and measurements using single individuals are susceptible to detection biases (Cook et al., 2012; Bates et al., 2015).

In the analysis phase, most marine climate change studies include only a single predictor - temperature to explain changes in distribution or phenology and thus do not explicitly consider other drivers of change (Brown et al., 2011). It is unknown whether studies that do not account for other potential anthropogenic drivers, such as fishing, eutrophication and habitat loss, could lead to higher estimates of impacts of climate change. For example, an investigation of changes in the distribution of North Sea cod showed fishing pressure explains part of the observed biological changes (Engelhard et al., 2014).

A greater understanding of how different methodological approaches affect the detection of observed responses to climate change will benefit climate change ecology in four main ways. First, studies that analyse existing data sets to test for climate impacts need to account for historical choices made about field data collection that could limit the ability to detect species' responses to climate change. For instance, uneven sampling effort along coasts means museum records of species occurrences may misrepresent historical range boundaries (Shoo et al., 2006; Przeslawski et al., 2012). Second, many regional studies compare rates of change with other studies in their discussions of how ecological traits influence a species' response (e.g. Richardson, 2008). Comparisons of change may also need to consider differences in methodological approaches across studies, such as how occurrence data are used to estimate ranges. Third, new time series are being initiated with the aim of measuring future distribution and phenology change (e.g. Robinson et al., 2015). Greater understanding of how different methodological approaches can affect measured responses to climate change could assist the design of new time series. For instance, inconsistent sampling effort through time may bias measured rates of change (Bates et al., 2015). Finally, meta-analyses of existing studies will produce more accurate estimates if they standardize for study differences, or constrain comparisons to be among studies with similar methods (Parmesan, 2007; Przeslawski et al., 2012). For instance, differences in rates of range shifts among European butterflies, birds and plants could be a consequence of taxonomic identity, geographical biases or differences in the metrics used (Parmesan \& Yohe, 2003).

Here, we examine the potential causes of variability in observed marine species distribution and phenology responses to climate change using a meta-analysis. First, we ask whether measurements of distribution and phenology change from the peer-reviewed literature are representative of different taxa, oceans and methodological approaches. Then, we conduct a metaanalysis on rates of response, to ask how ecological traits, study design and measurement approaches influence rates of distribution and phenology change. This enables us to ask how study measurement approaches may bias measured rates of change and affect inferences about the biological drivers of change. Finally, we investigate how different measurement approaches 
are used for different taxa and discuss the implications of measurement biases on the global understanding of climate change responses.

\section{Materials and methods}

\section{Database}

We analysed the database of peer-reviewed studies of observed impacts of climate change on marine organisms compiled by Poloczanska et al. (2013). We used a subset of 61 studies where rates of range change in phenology or distribution were reported or could be calculated and updated the database with a further 18 studies published in 2011-2014, for a total of 79 studies (Tables S1 and S2). In the original database and the update, we made every effort to include every data set and study that met our criteria; thus, we believe this to be a comprehensive data set rather than a small subset of available data.

Three criteria were applied for inclusion of peer-reviewed studies in the database: (1) authors must have inferred or directly tested for trends in marine ecological and climate variables from field observations; (2) observations spanned at least 19 years; and (3) studies included data after 1990. Studies relying purely on modelling or experimental data were excluded. At least twenty years of data is a common cut-off used in syntheses of climate change impacts (Rosenzweig et al., 2008). This length of time gives confidence that biological changes might be driven by long-term climate change rather than yearly climate variability. We chose to use nineteen years as the minimum time span, as several large studies had durations of 19 years. Requiring studies to also have data after 1990 ensures that there are observations in recent decades when the anthropogenic climate signal has been strongest.

Inclusion of all peer-reviewed studies resulted in some pseudoreplication of observations. In some cases, multiple studies analysed the same raw data set, and in other cases, multiple metrics of change were reported for a single species in a region. In such cases, only the most recent study for a given data set was included in the main database. Non-independent observations were removed from the database, using a decision tree based on data and analysis quality (Poloczanska et al., 2013). Following this process, 47 distribution studies with 485 observations of change and 32 phenology studies with 156 observations of change remained and were included in our analyses.

\section{Analysis of rates of change}

First, we summarize measurements of distribution and phenology change by methodological attributes, taxon, latitude, and for phenology, season. We then conducted analyses to examine how rates of change were influenced by ecological traits and methodological approaches. Rates of change, in kilometres per decade or days per decade, were obtained from individual studies in the database, either directly as reported in the text, calculated from figures, or by contacting the study's authors. Distribution changes were recorded as positive where they were consistent with a response to climate change (generally polewards, but see Burrows et al., 2011) and negative if they were not consistent with climate change. Phenology changes were recorded as negative for shifts to earlier dates and positive for shifts to later dates.

We related rates of change to a suite of predictors using a generalized linear modelling approach. The response variables were the rate of change in either distribution or phenology. Predictors were divided into two categories: methodological approaches and ecological traits. For methodological attributes, we considered the frequency of sampling (continuous, irregular or comparison of two periods). Fewer sampling points for studies that compare two periods of time (e.g. repeating a historical survey) may mean less accurate (either higher or lower) rates of change, because intervening fluctuations are ignored. We considered the number of species in a study; studies reporting on more species were expected to have lower rates of change because they are less likely to be influenced as strongly by publication bias (Parmesan, 2007). We also considered whether non-climatic drivers of change were accounted for in the study. We expect slower rates of change in studies with non-climatic drivers because confounding influences on the response could explain some of the observed variation. For distribution, we considered whether rates were generated from abundance (or relative abundance) metrics or from present data on species occurrence at sites. Occurrence-based data were expected to have higher rates of change because they are more susceptible to the outlying influences of a single individual. Similarly for phenology, we considered whether the magnitude of change in timing was related to whether the measure was an abundance metric, or the timing of the most extreme individual (e.g. first or last arrival - the temporal analogue of single sightings on a range edge). Extreme timings were expected to have higher rates of change (Moussus et al., 2010).

For ecological traits, we considered whether life-history development type (direct development with no larval phase, meroplanktonic, planktonic), exploitation status (commercially targeted or not), the mean latitude of the observations for a species and the depth range of the organism (coastal, demersal or pelagic) could influence the rates of change measured (Poloczanska et al., 2013). For the depth range, species were classified based on the life-history stage that was studied and coastal species were those constrained to terrestrial (seabirds), intertidal (e.g. barnacles) or near-shore (e.g. anemones) habitats (Poloczanska et al., 2013). Additionally, for distribution, we considered whether the measurement was made for a leading (poleward) or trailing (equatorward) edge, or for the population centre. For phenology, we considered the season of the measurement. Where available, ecological traits were extracted during the review of each study, based on what the individual studies reported. Latitude, range edge and season could also be considered as methodological approaches; however, we classified them as ecological traits because ecological expectations can be given for their effect on climate responses (Davis et al., 2010). 
We also included climate predictors in analyses: the velocity of climate change ( $\mathrm{km}$ per decade) for distribution and seasonal shift (days per decade) for phenology. The climate predictors measure the expected rate of response if species are tracking thermal niches in space and time (Loarie et al., 2009; Burrows et al., 2011). The indices were calculated for each study following Burrows et al. (2011). In brief, we used a global database of monthly sea surface temperatures, at a resolution of $1^{\circ}$ (Rayner et al., 2003). First, we spatially matched every species observation to a grid square. Where studies had a larger extent than a single grid square, we matched them to a grid square at the centroid of a study's location, or the nearest ocean cell if the centroid fell on land. We then calculated the decadal rate of temperature change for each study's centroid using linear regressions of mean annual temperatures. The time period was chosen to match each study's duration. For distribution, we additionally calculated the spatial gradient in temperature $\left({ }^{\circ} \mathrm{C} \mathrm{km}{ }^{-1}\right)$ by taking the mean temperature in each grid square across the each study's time span, then calculating the spatial gradient in temperature as the vector sum of the north-south and east-west components of spatial change. For phenology, we additionally calculated the seasonal gradient in temperature over the dates of each study, as the mean of the monthly temperature differences over each season. Velocities were then calculated as the spatial or seasonal gradient in temperature divided by the interannual trend (Burrows et al., 2011).

We used linear mixed-effects models to relate rates of change to the suite of methodological and ecological predictors ['LME4' package in the $\mathrm{R}$ programming language (Bates et al., 2014b)]. Taxon was treated as a random effect because our main interest was to characterize rates of change by ecological traits and measurement type, which are correlated with taxa. For distribution and phenology, we developed separate full models including all physical, ecological and measurement predictors. Models were simplified using a stepwise selection process, removing the variable that caused the greatest reduction in the Bayes information criteria (BIC) at each step. The selection process stopped when no further predictors could be removed without increasing the BIC. We chose to use the BIC over the Akaike information criteria because the BIC is less likely to include spurious predictors and it controls for sample size (Burnham \& Anderson, 2002). Thus, the final models could be considered conservative in that they include only the strongest predictors of rates of response to climate change.

We plotted effect sizes for predictors included in the final models on term plots. Term plots illustrate the modelled effects of a predictor relative to the mean of all predicted values. Term plots are an appropriate way to display effect sizes when there is no control treatment, because comparisons can be made across all predictions. A positive value for an effect on the term plot indicates that a predictor increases the rate of an organism's response to climate change. A negative value indicates the effect slows an organism's response to climate change. Terms were presented with confidence intervals, which were estimated using bootstrapping [using the 'ВоОт' package in R, see Canty \& Ripley (2014)].
We estimated the relative importance of methodological approaches when compared to ecological traits by comparing the proportions of variance explained by each set of predictors. We estimated variance explained by either measurement approaches or ecological traits as the difference between the marginal $R^{2}$ statistic (Nakagawa \& Schielzeth, 2013) for the model with all significant predictors and a model without the respective variables.

Following the analysis, we examined in more detail how inferences drawn from the analysis of the database may be influenced by the available studies. Specifically, we plot the frequency of measurement for the variables that were significant predictors of distribution and phenology change by taxa.

\section{Results}

\section{Summary of distribution and phenology observations}

Across all the distribution and phenology studies, there were many biases in study attributes and methodologies (Fig. 1). Of 47 distribution studies and 32 phenology studies, only 15 and 6, respectively, had more than one species, although only 2 distribution studies and no phenology studies had $>10$ species (Fig. 1a). Of 485 distribution measurements, occurrence-based measures of distribution were slightly more common than abundance-based measures (Fig. 1b). For 156 phenology observations, abundance-based measures were more common than measurements of extreme individuals (Fig. 1b). 38\% of distribution responses compared two points in time, whereas $85 \%$ of phenology responses were measured from continuous time series (Fig. 1c). Most distribution and phenology data were collected in mid-to-high latitudes, with phenology records, in particular biased towards the Northern Hemisphere and a remarkable paucity of observations for tropical species (Fig. 1d). There was considerable bias in taxonomic representation; $41 \%$ of distribution records were for bony fish and 19\% for benthic algae (Fig. 1e), whereas $33 \%$ of phenology records were for seabirds and $51 \%$ for phyto- and zooplankton, which were both poorly represented in distribution records (3\% and $1 \%$, respectively). Most benthic taxa had distribution observations, but few phenology observations. Measurements of phenology tended to be made in spring and summer, but rarely in autumn or winter (Fig. 1f).

\section{Effects of ecological traits and methodological attributes on distribution rates of change}

The final model for the rate of distribution change included one ecological trait and two methodological approaches (Table 1, Fig. 2). Estimates of change 

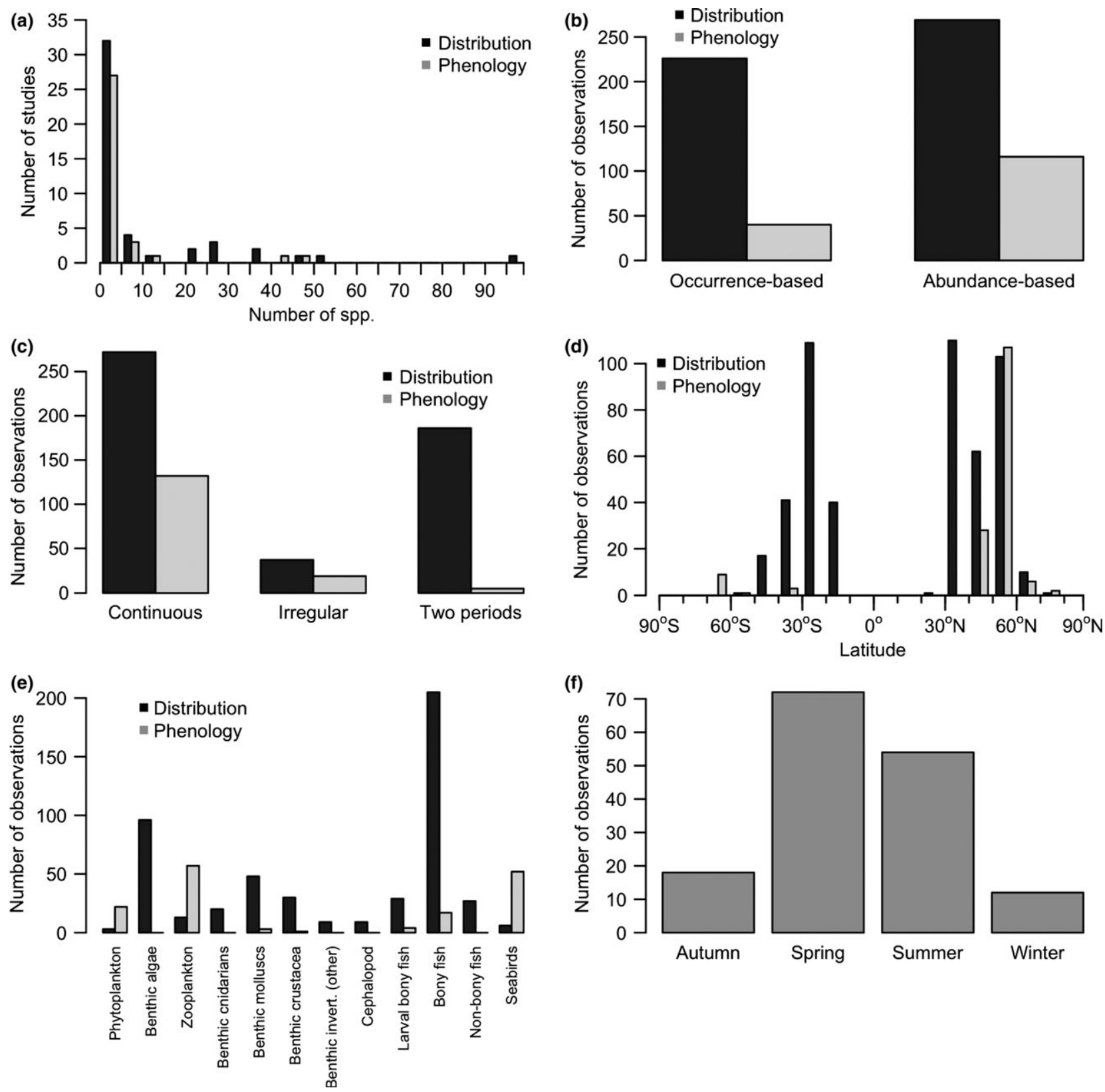

Fig. 1 Frequency of different factors in studies of distribution and phenology: (a) number of species in a study; (b) occurrence-based or abundance-based measures of distribution and phenology; (c) sampling frequency; (d) latitude; (e) broad taxonomic groupings; and (f) season, for phenology.

derived from irregular time series or those that were calculated by comparing two points in time tended to be faster than continuous time series (Fig. 2). Occurrence-based measures of distribution change were also faster than abundance-based measures. Demersal and pelagic species moved faster than coastal species (intertidal species and seabirds). A model including these top-ranked predictors suggests that phytoplankton have changed distributions faster than other taxa, whereas benthic cnidarians and seabirds have changed the slowest (Fig. 2). The reduced model explained $28 \%$ of the variance, with methodological approaches (sampling frequency and type of measure) accounting for $22 \%$ of the variation in rates of change and ecological variables (depth zone) accounting for 7.8\% (there was shared variance across methodological and ecological variables, so the individual variables did not add up to the total variance explained).

Ecological traits that were excluded from the final model included the range edge, which was not a 
Table 1 Analysis of rates of distribution and phenology change, with the $\Delta \mathrm{BIC}$ calculated by adding (for non-significant variables) or removing (for significant variables) each variable from the reduced model

\begin{tabular}{lllr}
\hline Factor & Variable class & Distribution data set $\Delta$ BIC & Phenology data set $\Delta$ BIC \\
\hline Abundance-/occurrence-based measure & Methodological approach & 23 & -4.5 \\
Data sampling frequency & Methodological approach & 64 & -9.7 \\
Non-climatic factors considered & Methodological approach & -6.1 & 2.8 \\
No. of spp. in study & Methodological approach & -5.4 & -4.0 \\
Time span of study & Methodological approach & -5.4 & 0.2 \\
Depth category & Ecological trait & 47 & -9.5 \\
Exploitation status & Ecological trait & -4.0 & -4.9 \\
Latitude & Ecological trait & -1.2 & 2.8 \\
Planktonic larval dispersal type & Ecological trait & -2.5 & -5.6 \\
Range edge/centre & Ecological trait & -6.9 & N.A. \\
Season & Ecological trait & N.A. & 10.4 \\
Velocity of climate change & Ecological trait & -2.2 & -4.6 \\
\hline
\end{tabular}

Variables with negative $\triangle \mathrm{BIC}$ were not included in the reduced models. N.A., not applicable.

(a)

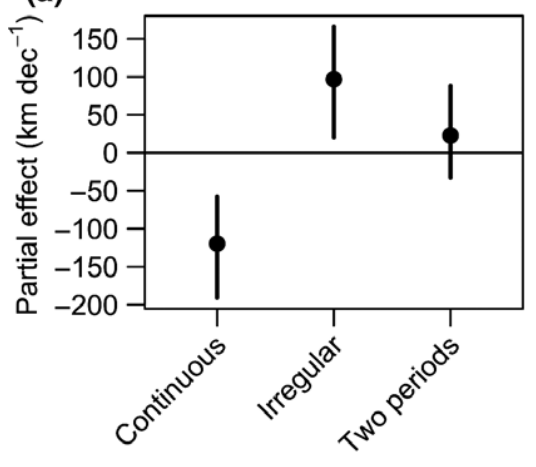

(c)

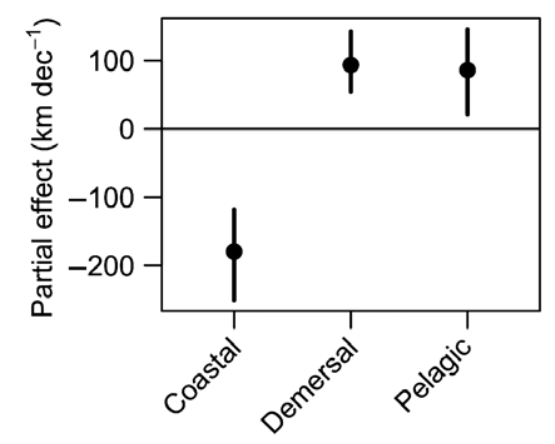

(b)

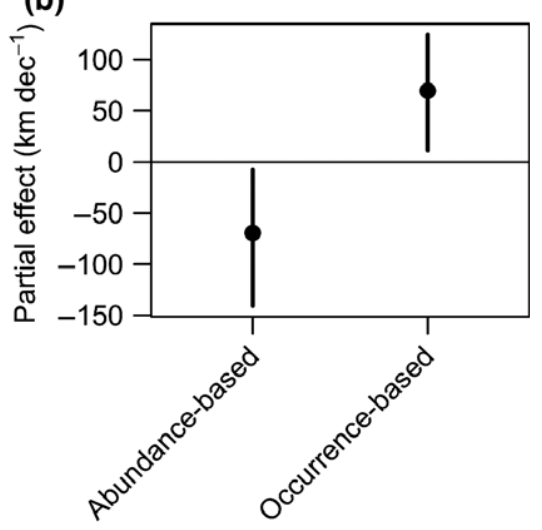

(d)

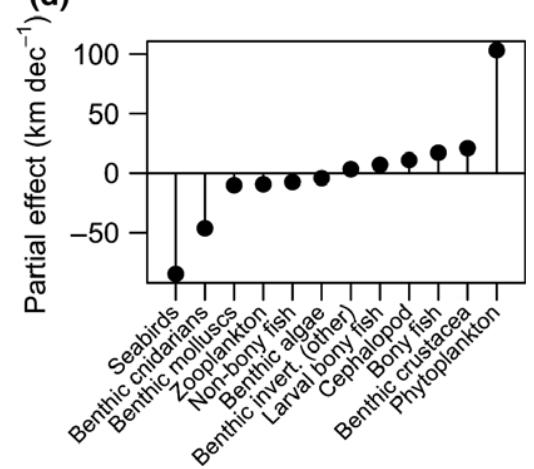

Fig. 2 Term plot for the analysis of rates of change in distribution using the final mixed-effects model, showing the final model (selected using BIC, full model BIC $=5713$, reduced model BIC $=5686$ ). For the fixed effects, points indicate mean predicted effects and bars represent $95 \%$ confidence intervals. Taxa were treated as a random effect. Effects are standardized to the overall mean, so positive effects indicate a tendency towards distribution change that is greater and consistent with climate change, whereas negative effects indicate a tendency towards smaller changes or changes that are not consistent with warming (although those two cannot be distinguished in this figure). Note the varying scales for the $y$-axes; larger ranges indicate larger effect sizes.

parsimonious predictor of distribution change (Table 1). The velocity of climate change was also excluded from the final model, while the model estimated a positive effect of higher velocities on distribution change, the velocity effect was weak. The number of species in each study, a methodological variable, was also 
excluded from the final model, suggesting there is no strong effect of publication bias in this analysis.

\section{Effects of ecological traits and methodological attributes} on phenology rates of change

The final reduced model for phenology change explained $14 \%$ of the variance in the data and included four factors, time span, season, inclusion of non-climatic factors and latitude (Table 1, Fig. 3). It excluded sampling frequency, depth range, life-history development type, exploitation status, seasonal climate shift, measurement type and the number of species in a study. Studies that used shorter time series were more likely to report earlier timings, suggesting a slight publication bias, although the effect size was small. However, counter to our expectations, studies that considered non-climatic factors estimated faster rates of change than studies that did not. Phenological events at midlatitudes were more likely to be slower than those at higher latitudes. The phenology of autumnal events typically shifted later, rather than earlier. Overall, the effects of ecological traits and methodological attributes were small ( $8 \%$ and $4 \%$ of the variance, respectively) compared to the random effect of taxa on rates of change. Larval bony fish were most likely to be shifting events earlier, whereas seabirds had small changes in phenology or were likely to be shifting later.

\section{Differences in how responses are measured across taxa}

Next, we examined how observations are distributed across taxa, ecological traits and methodological approaches to explore the representativeness of climate research. We focus on the ecological traits and methodological approaches that were significant predictors of rates of change. Importantly, not all taxa had measurements with every methodological approach (Figs 4 and 5). Lack of measurements indicates caution should be taken when extrapolating the results of meta-analyses to poorly sampled taxa.

For distribution, occurrence-based measures (that tended to report larger distribution changes) predominated over abundance-based measures. Most abun-
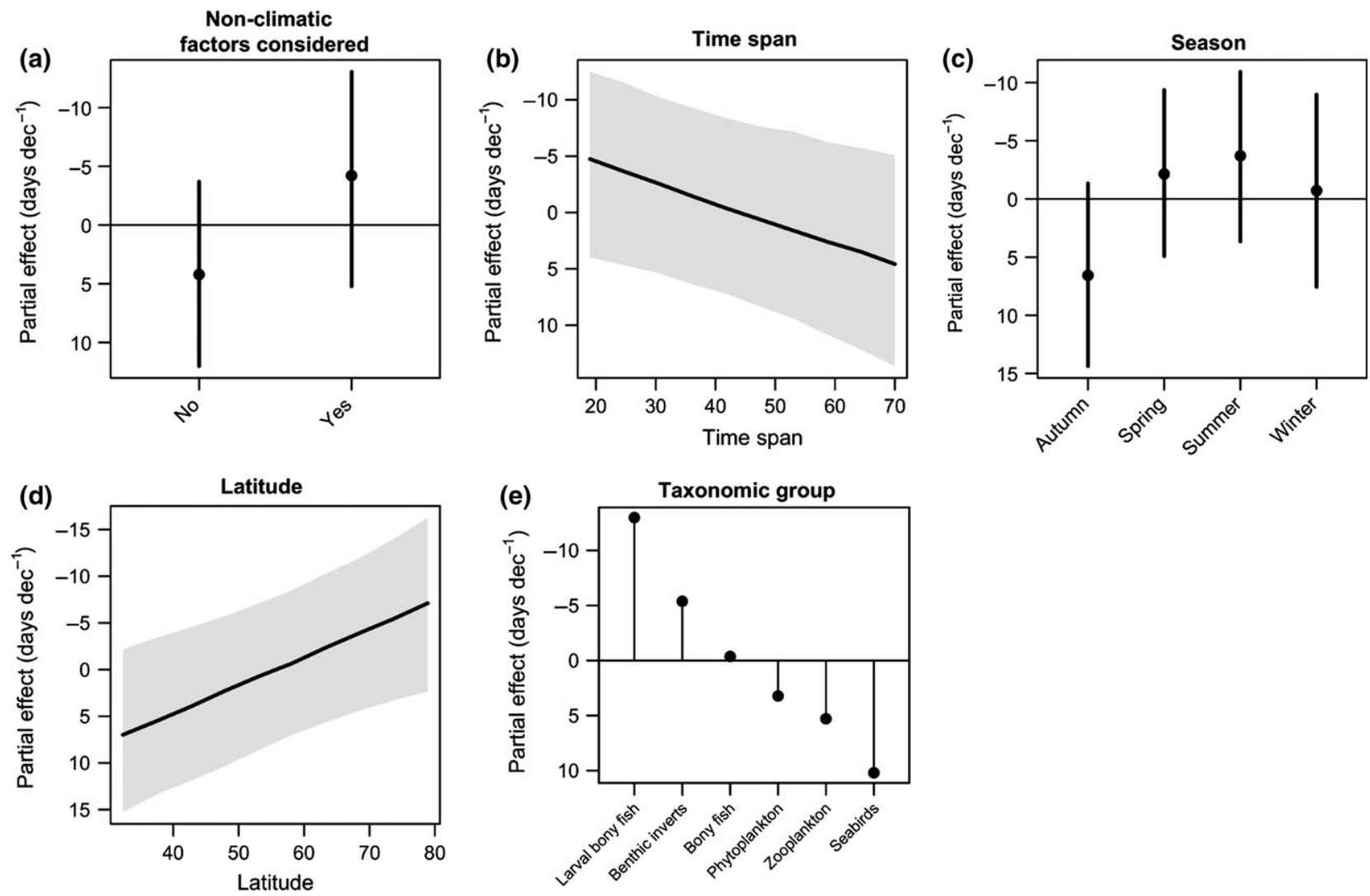

Fig. 3 Term plot for the analysis of rates of change in phenology using the final mixed-effects model, showing the final model (selected using BIC, full model BIC $=1153$, reduced model BIC $=1112$ ). Points indicate mean predicted effects and bars the $95 \%$ confidence intervals. Taxa were treated as a random effect. Effects are standardized to the overall mean, so negative effects (upwards on the $y$-axis) indicate a tendency towards phenology change that is earlier and consistent with climate change, whereas positive effects indicate a tendency towards smaller date changes or changes that are not consistent with warming. Note the varying scales for the $y$-axes. 


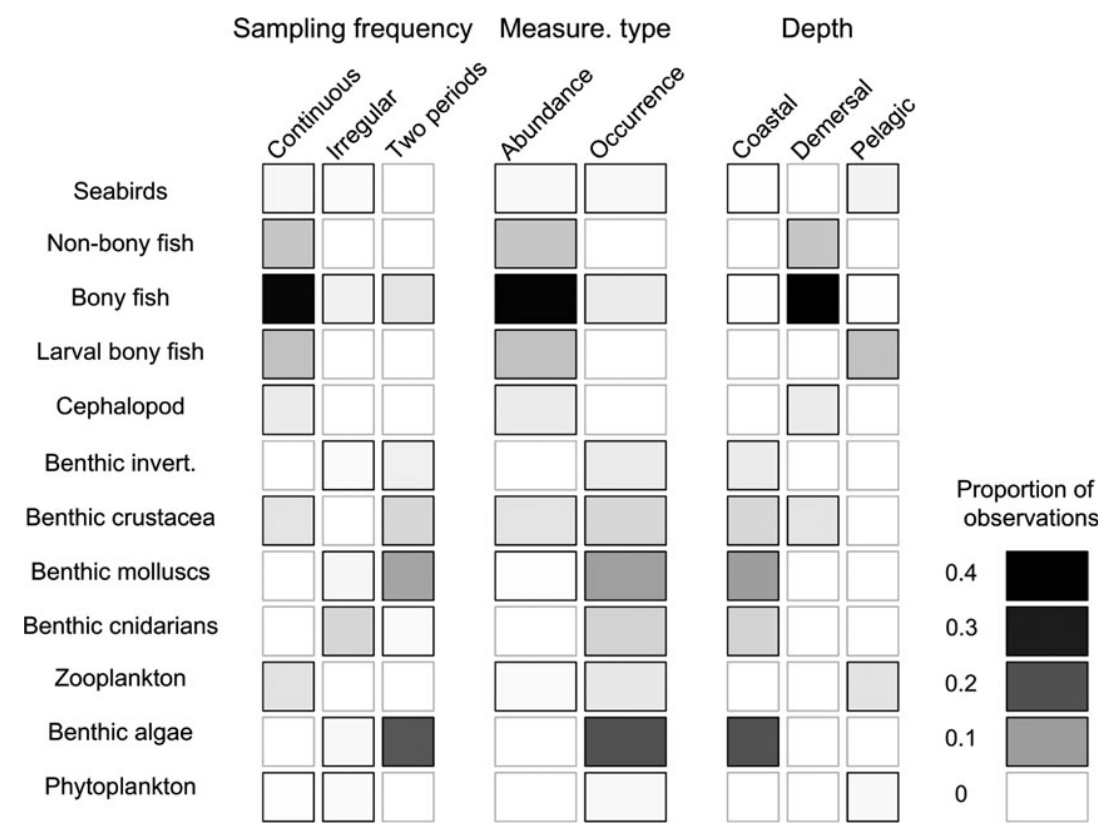

Fig. 4 Proportion of distribution observations by taxa and each covariate used in the final model for distribution rate of change. The maximum proportion of observations in any category was 0.4 .

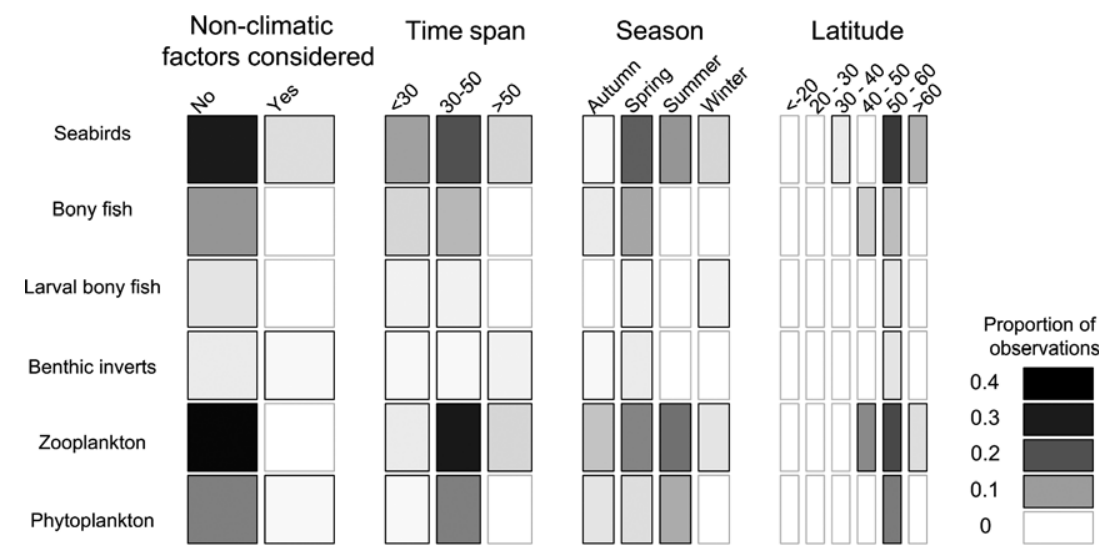

Fig. 5 Proportion of phenology observations by taxa and each covariate used in the final model for distribution rate of change. The maximum proportion of observations in any category was 0.4 .

dance-based measures came from fish and larval fish studies, which typically use nets to sample species in fishery-related surveys (Fig. 4). Occurrence-based measures were predominant in other taxa. Fishery-related surveys also had many more continuous time series, rather than comparisons of two points in time. In particular, benthic molluscs, benthic cnidarians, macroalgae and other invertebrates had no continuous time series.

Although measurements of phenology change were faster in studies that considered non-climatic factors (Fig. 3a), there were very few studies (only 7) that considered non-climatic factors in their analyses (Fig. 5). Observations that come from studies that considered non-climatic factors were mostly for seabirds, but there was also a small proportion for phytoplankton and benthic crustaceans. All taxa were represented in data sets with time spans of up to 50 years. Autumn and spring were also well represented; however, many taxa did not have phenological measurements in summer and winter. Most phenology records for most species were at latitudes $>40^{\circ}$, and only seabirds had measurements closer to the equator.

\section{Discussion}

The methodology used to standardize studies for metaanalyses can have considerable effects on estimates for 
rates of response to climate change (Parmesan, 2007; Przeslawski et al., 2012). Typically, meta-analyses exclude some studies to achieve consistency, such as excluding studies of single species to avoid publication bias (Parmesan \& Yohe, 2003; Parmesan, 2007; Poloczanska et al., 2013). Rather than excluding studies, we used linear models to standardize for differences in approaches across studies. By including studies that used different methods to measure change, we have quantified the size and direction of methodological effects on estimates of distribution and phenology responses to climate change. We found methodological differences explained $22 \%$ of the variation in range shifts, more than the $7.8 \%$ of the variation explained by ecological traits. For phenology change, methodological approaches accounted for $4 \%$ of the variation in measurements, whereas $8 \%$ of the variation was explained by ecological traits. Our study bolsters other recent findings that different approaches to observing a single pattern (e.g. a geographical distribution) can lead to different estimates of change over time (Moussus et al., 2010; Wolkovich et al., 2012; Bates et al., 2015). Below, we discuss the implications of differences in study design and biological traits on estimated rates of change.

\section{Study design}

We found studies comparing two points in time or using irregularly sampled time series measured greater rates of change than studies using continuous time series. Continuous time series should quantify rates of change more accurately than infrequent sampling, because infrequent samples confound short-term variability with long-term trends (Moussus et al., 2010; Brown et al., 2011). Further, range shifts in response to climate change can be confounded by inconsistent sampling effort when a species is unlikely to be detected at every sampling event (Bates et al., 2015). Studies in our database that had infrequent sampling often resurveyed sites that were sampled historically, so our result may also suggest some publication and study site-selection bias towards places where ecological change has been greatest.

Historical comparisons (i.e. resurveying) are an important way to create long-term studies, where there has not been funding to support long-term sampling (e.g. Southward et al., 2004; Przeslawski et al., 2012; Robinson et al., 2015). Studies of fish were more likely to have continuous time series, presumably because of their economic importance, whereas observations for other taxa often came from comparisons of two points in time. We encourage authors to look for and publish resurveys of historical sampling, regardless of whether there have been considerable changes in distribution, to help overcome potential publication biases. Efforts to digitize and publish historical data sets (e.g. Engelhard et al., 2014), combined with the growth of data journals with the mandate that data generated using public funds must be made available, may lead to many such data sets surfacing in the future, providing a richer and less biased basis to assess responses to climate change.

Abundance-based estimates of distribution change were slower than occurrence-based measures. Occurrence-based measures can be influenced by responses of single individuals or by detectability of a species, so we expected their observed rate of change to be greater (Brown et al., 2011; Bates et al., 2015). Occurrence-based measures may be more likely to detect change, but also more susceptible to spurious affects. Occurrence and abundance measures also reflect different aspects of distribution change (Bates et al., 2014a). Occurrencebased measures are sensitive to range expansion, whereas abundance-based measures better reflect population establishment. As such, our analysis suggests that population establishment occurs much more slowly than range expansion - taking the difference in rate of change between abundance and occurrencebased measures, the analysis suggests on average a lag of about $140 \mathrm{~km}$ per decade, which is of greater magnitude than rates of change in individual species' range centres (Poloczanska et al., 2013). Further, this result indicates that caution should be taken when extrapolating rates of change across different locations. Ranges may expand rapidly as few individuals of a species occupy areas it was previously absent from, but population establishment may follow more slowly (Bates et al., 2014a). The pattern of range expansion and population establishment is particularly important when managing ecosystems dynamically as communities move into novel configurations with climate change (Graham et al., 2014).

Studies with single species or short time series are often excluded from meta-analyses because of perceived publication bias towards publishing results consistent with climate change (Parmesan \& Yohe, 2003; Parmesan, 2007). Based on the analysis of length of study as a predictor, we found no publication bias in distribution studies and only a small bias in phenology studies. Publication bias may be less prevalent in marine than terrestrial studies because overall there are more multispecies studies in marine ecosystems where sampling methods tend to collect numerous organisms (e.g. fish and plankton) by nets (Richardson et al., 2012).

We found that inclusion of non-climatic factors in the analysis increased the estimated rates of phenology change, but had no effect on rates of distribution 
change. However, few studies included non-climatic factors, so further investigation of how climate responses interact with factors such as fishing pressure and eutrophication is important. Phenology is sensitive to multiple human impacts, and it may be that in the studies analysed here, those impacts are also causing seasonal timings to occur earlier. Given the paucity of studies, further work is required to assess the interaction between climate change and other variables (Parmesan et al., 2013).

\section{Ecological traits}

We found that pelagic and demersal species tended to move faster than coastal (inshore) species. Coastal species such as kelps and rocky shore invertebrates may be less able to track warming because their distributions are restricted to the coast, and hence subject to non-climatic biogeographical barriers to simple latitudinal shifts (Broitman et al., 2008). For instance, limited availability of hard substrates on Australia's east coast may limit poleward migration of rocky intertidal organisms (Poloczanska et al., 2011).

Contrary to previous studies for terrestrial and marine ecosystems, we did not find that leading edges moved faster than trailing edges (Parmesan et al., 1999; Sunday et al., 2012; Poloczanska et al., 2013). Range edges are more likely to be detected with occurrencebased measures, because species tend to be rarer at their range edges (Sagarin et al., 2006); therefore, the measurement type and the position of measurements within a species' range may be confounded. In our analysis, sufficient measurements of species abundance at range edges were available to distinguish the effects of range position and measurement type on the rate of distribution shift. We found that occurrence-based measurements were generally faster than abundance-based measurements. Thus, if measurement differences are not accounted for, studies may overestimate the rate of shift at range edges, because of the bias towards measuring edges using single individuals.

\section{Gaps in climate change ecology studies}

Our analyses revealed that many gaps remain in our understanding of distribution and phenology responses to climate change. Gaps are a consequence of not only geographical biases in sampling effort, but also how different taxa are studied. In particular, a strong geographical bias exists towards temperate regions, where data collection efforts have historically been the greatest. Tropical regions, grossly under-represented in current studies (Lenoir \& Svenning, 2015), are expected to display some of the highest rates of distribution change, particularly in marine systems (Burrows et al., 2014), and the distributions of tropical taxa may be particularly susceptible to warming (Sunday et al., 2012; Molinos et al., 2015). Moreover, the subtropical and tropical ocean represents $\sim 70 \%$ of the global ocean surface, implying that the current paucity of studies of distribution and phenology shifts in the tropics affects our capacity to extrapolate available data to global rates. Global rates of distribution change estimated here are therefore likely lower than those that would be inferred if the studies were randomly distributed across latitudes.

There were few long-term phenology studies in the tropics. While seasonality in temperature is weaker in the tropics and our results suggest phenology change is slower towards lower latitudes, warming can still drive temporal changes in species abundance, for instance blooms of dangerous jellyfish (Jacups, 2010). In addition to warming, seasonality along tropical coastlines can be driven by precipitation (Van Schaik et al., 1993; Chambers et al., 2013). For example, the timing of juvenile prawn migrations from rivers to the ocean is related to cumulative rainfall in tropical river basins (Staples \& Vance, 1986). Phenological response to precipitation is more difficult to predict than warmingrelated responses because species may shift earlier or later (Chambers et al., 2013). Impacts of climate change on tropical seasonality have historically been neglected and warrant further studies.

There were systematic differences in types of observations across taxa, which suggests that we have major gaps in our understanding of climate impacts. For instance, seabird studies that measured changes in phenology with climate change were common, whereas there were only two seabird studies of distribution that met the criteria for inclusion in our database. This is likely to be because seabirds are most easily sampled at breeding colonies where there have been long-standing monitoring programmes (e.g. Barbraud \& Weimerskirch, 2006), rather than during their extensive foraging forays. In the future, the extensive and ongoing tracking information collected using satellite tags should provide long-term information on distribution shifts in feeding distributions and potentially on shifts in breeding sites. In contrast to seabird studies, studies of fish distribution were common, and studies of fish phenology are rare. Fish studies in the database often used fisheries data sets for analysing climate patterns. Fishery-related surveys are largescale, regular (usually annual) surveys of abundance indices. They are typically annual, so they cannot be used to measure phenology, but they do often cover large geographical areas, so they are very suitable for distribution studies. 
Few observations of marine phenology were available from autumn or winter, a potential temporal bias that also occurs in terrestrial studies of phenology (Gallinat et al., 2015). The lack of data on phenology from autumn and winter could partially reflect the fact that many species perform many of their most important processes (e.g. feeding and reproduction) predominantly in spring and summer. There are also many more spring than autumn observations for terrestrial ecosystems (Parmesan \& Yohe, 2003). But unlike marine systems, terrestrial systems do have a few very long-term (e.g. grape harvests over 800 years (Menzel, 2005)) and iconic (e.g. fall colour indexes in New England, USA (Gallinat et al., 2015)) autumn measurements. We found evidence that autumnal events were shifting later, rather than earlier, which is consistent with lengthening seasons. Measuring autumn phenology in higher latitudes is therefore an important knowledge gap in both marine and terrestrial systems. Because of this gap, we have little information on how growing seasons may be extended by warming (for an example see Moore et al., 2011).

\section{Recommendations for measuring change}

Based on the findings of our meta-analysis, we make several recommendations for measuring responses of organisms to climate change when analysing past studies of climate change impacts or designing new studies.

Reanalysis of existing time series. A critical question is whether the time series can be used to address the study's aim. For instance, a database of species occurrences across space and time can be used to examine colonization of new areas, but is more limited in supporting inferences about the establishment and persistence of new populations. Similarly for phenology, a time series of the most extreme individuals' breeding timing does not necessarily reflect significant change in a whole population, although changes in a few individuals may be an early warning for population-level change. Therefore, researchers should be careful to consider the potential limitations and biases in data when conducting reanalyses.

Covariates, particularly those not related to climate, are key to consider when analysing time series. A typical test is to ask whether warming is driving an observed change, with the null hypothesis being warming is not a factor. Greater consideration of other alternatives is important (Brown et al., 2011), including nonclimate drivers of distribution and phenology. For instance, changes in depth range of an organism could be driven by warming, but the potentially confounding effect of fishing pressure changing with depth should also be considered in such an analysis (Dulvy et al. 2008; Engelhard et al., 2014).

Broadening the scope of climate change studies to include other drivers will require greater accessibility of data on human impacts - such as fishing and eutrophication at appropriate time and space scales. Climate time series data are widely available as free downloads, whereas data on other drivers are often less easily available or do not exist at all. Efforts to share currently closed databases, such as those on fishing and efforts to collect more data, for instance using satellite images to map eutrophication, will enable better discrimination of climate from other signals and thus more robust climate attribution.

Qualitative comparisons with other studies. Qualitative comparisons among rates of change are common in regional or taxon-specific studies of climate change (e.g. Richardson, 2008). The usual aim of qualitative comparisons is to give context for an observed rate of change being faster or slower than typical and to speculate about the ecological or environmental drivers of a difference. However, differences will also be strongly influenced by measurement biases. Where possible, qualitative comparisons should be made with similar metrics used to measure observed change. For instance measurements of distribution based on occurrence at sites should not be compared with measurements based on abundance averages, which are typically slower. As the number of climate studies grows, it will become easier to compare studies that use similar methods.

Meta-analyses of species responses to climate change. It is especially important for meta-analyses of species responses across many studies to include the influence of different variables explicitly in a statistical model. Important covariates include measurement and ecological variables. Past studies have either ignored these differences (Poloczanska et al., 2013) or tried to remove non-similar studies (Parmesan \& Yohe, 2003; Parmesan, 2007; Przeslawski et al., 2012). While including additional measurement variables in analysis did not significantly change our results when compared to previous analyses (Poloczanska et al., 2013), it did shed important light on factors affecting distribution and phenology change. Removing studies from analyses focuses on a subset of potential biases (e.g. only including studies on multiple species or time series greater than a certain length) and is a blunt approach that does not consider other potential sources of bias (e.g. measurement type, latitude, non-climate factors). Thus, removing studies from analysis reduces the power to detect real biological effects and therefore should be avoided where possible. 
Design of new studies. Numerous time series are currently being started, with the aim of monitoring effects of climate change (e.g. Robinson et al., 2015). Our findings provide some advice on designing sampling protocols. The aims of measuring the time series should be explicitly defined and a protocol designed to address them. For example, a study that seeks to monitor invasion of pest species may focus on monitoring for occurrences, to obtain early warnings of range shifts. In contrast, a study that aims to detect ecologically significant might focus on monitoring abundance.

When designing a study, abundance-based measures therefore offer more explanatory power because they can be used to measure both colonization and establishment. Further, abundance-based measures will be less influenced by extreme occurrences of individuals, so may be a more robust measure of change (Brown et al., 2011). However, there may be trade-offs in sampling effort that warrant consideration. Occurrences are cheaper to measure than abundances, particularly for rare species, so occurrence surveys may cover larger areas and a greater range of species than abundance-based surveys could for the same cost. Further, occurrence-based measures are easier to obtain from non-experts, such as through citizen science programmes or from observations from fishers (Robinson et al., 2015). Occurrence-based measures could therefore provide a more useful early warning of invasion of new species, but do not necessarily indicate establishment of a new population.

A common approach to detecting climate change impacts is to resurvey sites that had historical measurements of climate change. Such resurveys are important to fill data gaps, yet our results suggest some selection bias for sites with greater change, at least for distribution studies. It is important that resurvey sights that are selected randomly (or comprehensively) to provide a less biased global view of climate change impacts, for instance by systematically sampling across a species' entire range.

Large gaps remain in our knowledge of climate change responses in both terrestrial and marine systems. Key among these is that there is three times as much information on changes in distribution than on phenology in the ocean, whereas on land there is 100 times more information on phenology than on distribution change (comparing Poloczanska et al., 2013 with Rosenzweig et al., 2008). Expanding terrestrial studies of species' distribution change and marine studies of phenology change, particularly in autumn and winter, is important to give a comprehensive view of life's responses to climate change.

We found that measurement biases can have a substantial effect on inferences about the impacts of climate change on distribution and phenology. Greater consideration of measurement bias in climate impact studies will improve our understanding of how measurement methods affect observations and ultimately contribute to a more representative view of the impacts of climate change on organisms.

\section{Acknowledgements}

This work was conducted as part of the Understanding Marine Biological Impacts of Climate Change Working Group supported by the National Center for Ecological Analysis and Synthesis, a centre funded by NSF (Grant \#EF-0553768), University of California, Santa Barbara, California. CJB acknowledges a University of Queensland postdoctoral fellowship. JMP acknowledges the support from the Australian Research Council (CE0561435 and DP130100250). We are grateful to all the workers on climate change impacts which we have reviewed.

\section{References}

Barbraud C, Weimerskirch H (2006) Antarctic birds breed later in response to climate change. Proceedings of the National Academy of Sciences of the United States of America, 103, 6248-6251.

Bates AE, Pecl GT, Frusher S et al. (2014a) Defining and observing stages of climate-mediated range shifts in marine systems. Global Environmental Change, 26, 27-38.

Bates D, Maechler M, Bolker BM, Walker S (2014b) LME4: Linear mixed-effects models using Eigen and S4. ArXiv e-print; submitted to Journal of Statistical Software. Available at: http://arxiv.org/abs/1406.5823 (accessed 23 November 2015).

Bates AE, Bird TJ, Stuart-Smith RD et al. (2015) Distinguishing geographical range shifts from artefacts of detectability and sampling effort. Diversity and Distributions, 21, 13-22.

Beaugrand G, Kirby RR (2010) Climate, plankton and cod. Global Change Biology, 16, $1268-1280$.

Broitman B, Blanchette C, Menge B et al. (2008) Spatial and temporal patterns of invertebrate recruitment along the west coast of the United States. Ecological Monographs, 78, 403-421.

Brown CJ, Schoeman DS, Sydeman WJ et al. (2011) Quantitative approaches in climate change ecology. Global Change Biology, 17, 3697-3713.

Burnham KP, Anderson DR (2002) Model Selection and Multimodel Inference: A Practical Information-Theoretic Approach. Springer, New York.

Burrows MT, Schoeman DS, Buckley LB et al. (2011) The pace of shifting climate in marine and terrestrial ecosystems. Science, 334, 652-655.

Burrows MT, Schoeman DS, Richardson AJ et al. (2014) Geographical limits to species-range shifts are suggested by climate velocity. Nature, 507, 492-495.

Canty A, Ripley B (2014) воот: Bootstrap R (S-Plus) Functions. R package version 1.3-13.

Chambers LE, Altwegg R, Barbraud C et al. (2013) Phenological changes in the Southern Hemisphere. PLoS One, 8, e75514.

Cook BI, Wolkovich EM, Parmesan C (2012) Divergent responses to spring and winter warming drive community level flowering trends. Proceedings of the National Academy of Sciences, 109, 9000-9005.

Davis CC, Willis CG, Primack RB, Miller-Rushing AJ (2010) The importance of phylogeny to the study of phenological response to global climate change. Philosophica Transactions of the Royal Society B: Biological Sciences, 365, 3201-3213.

Dulvy NK, Rogers SI, Jennings S, Stelzenmuller V, Dye SR, Skjoldal HR (2008) Climate change and deepening of the North Sea fish assemblage: a biotic indicator of warming seas. Journal of Applied Ecology, 45, 1029-1039.

Edwards M, Richardson AJ (2004) Impact of climate change on marine pelagic phenology and trophic mismatch. Nature, 430, 881-884.

Engelhard GH, Righton DA, Pinnegar JK (2014) Climate change and fishing: a century of shifting distribution in North Sea cod. Global Change Biology, 20, 2473 2483.

Fitter A, Fitter R (2002) Rapid changes in flowering time in British plants. Science, 296, $1689-1691$

Gallinat AS, Primack RB, Wagner DL (2015) Autumn, the neglected season in climate change research. Trends in Ecology \& Evolution, 30, 169-176. 
Graham NA, Cinner JE, Norström AV, Nyström M (2014) Coral reefs as novel ecosystems: embracing new futures. Current Opinion in Environmental Sustainability, 7, 9 14.

Jacups SP (2010) Warmer waters in the northern territory - herald an earlier onset to the annual Chironex fleckeri Stinger season. EcoHealth, 7, 14-17.

Lenoir J, Svenning JC (2015) Climate-related range shifts - a global multidimensional synthesis and new research directions. Ecography, 38, 15-28.

Ling S, Johnson C, Ridgway K, Hobday A, Haddon M (2008) Climate-driven range extension of a sea urchin: inferring future trends by analysis of recent population dynamics. Global Change Biology, 15, 719-731.

Loarie SR, Duffy PB, Hamilton H, Asner GP, Field CB, Ackerly DD (2009) The velocity of climate change. Nature, 462, 1052-1055.

Menzel A (2005) A 500 year pheno-climatological view on the 2003 heatwave in Europe assessed by grape harvest dates. Meteorologische Zeitschrift, 14, 75-77.

Molinos JG, Halpern BS, Schoeman DS et al. (2015) Climate velocity and the future global redistribution of marine biodiversity. Nature Climate Change. doi: 10.1038/ nclimate2769.

Moore PJ, Thompson RC, Hawkins SJ (2011) Phenological changes in intertidal conspecific gastropods in response to climate warming. Global Change Biology, 17, 709 719 .

Moussus JP, Julliard R, Jiguet F (2010) Featuring 10 phenological estimators using simulated data. Methods in Ecology and Evolution, 1, 140-150.

Nakagawa S, Schielzeth H (2013) A general and simple method for obtaining R2 from generalized linear mixed-effects models. Methods in Ecology and Evolution, 4, 133 142

Parmesan C (2007) Influences of species, latitudes and methodologies on estimates of phenological response to global warming. Global Change Biology, 13, 1860-1872.

Parmesan C, Yohe G (2003) A globally coherent fingerprint of climate change impacts across natural systems. Nature, 421, 37-42.

Parmesan C, Ryrholm N, Stefanescu C et al. (1999) Poleward shifts in geographical ranges of butterfly species associated with regional warming. Nature, 399, 579-583.

Parmesan C, Burrows MT, Duarte CM, Poloczanska ES, Richardson AJ, Schoeman DS, Singer MC (2013) Beyond climate change attribution in conservation and ecological research. Ecology Letters, 16, 58-71.

Perry AL, Low PJ, Ellis JR, Reynolds JD (2005) Climate change and distribution shifts in marine fishes. Science, 308, 1912-1915.

Pinsky ML, Worm B, Fogarty MJ, Sarmiento JL, Levin SA (2013) Marine taxa track local climate velocities. Science, 341, 1239-1242.

Poloczanska ES, Smith S, Fauconnet L, Healy J, Tibbetts IR, Burrows MT, Richardson AJ (2011) Little change in the distribution of rocky shore faunal communities on the Australian east coast after 50 years of rapid warming. Journal of Experimental Marine Biology and Ecology, 400, 145-154.

Poloczanska ES, Brown CJ, Sydeman WJ et al. (2013) Global imprint of climate change on marine life. Nature Climate Change, 3, 919-925.

Przeslawski R, Falkner I, Ashcroft MB, Hutchings P (2012) Using rigorous selection criteria to investigate marine range shifts. Estuarine, Coastal and Shelf Science, 113, 205-212.
Rayner N, Parker DE, Horton E et al. (2003) Global analyses of sea surface temperature, sea ice, and night marine air temperature since the late nineteenth century. Journal of Geophysical Research: Atmospheres, 1984-2012, 108.

Richardson AJ (2008) In hot water: zooplankton and climate change. ICES Journal of Marine Science: Journal du Conseil, 65, 279-295.

Richardson AJ, Brown CJ, Brander K et al. (2012) Climate change and marine life. Biology Letters, 8, 907-909.

Robinson L, Gledhill D, Moltschaniwskyj N et al. (2015) Rapid assessment of an ocean warming hotspot reveals "high" confidence in potential species' range extensions. Global Environmental Change, 31, 28-37.

Rosenzweig C, Karoly D, Vicarelli M et al. (2008) Attributing physical and biological impacts to anthropogenic climate change. Nature, 453, 353-357.

Sagarin RD, Gaines SD, Gaylord B (2006) Moving beyond assumptions to understand abundance distributions across the ranges of species. Trends in Ecology \& Evolution, 21, 524-530.

Shoo LP, Williams SE, Hero J-M (2006) Detecting climate change induced range shifts: where and how should we be looking? Austral Ecology, 31, 22-29.

Southward AJ, Langmead O, Hardman-Mountford NJ et al. (2004) Long-term oceanographic and ecological research in the western English Channel. In: Advances in Marine Biology, Vol 47 (eds Southward AJ, Tyler PA, Young CM, Fuiman LA), pp. 1-105. Academic Press, Cambridge.

Staples D, Vance D (1986) Emigration of juvenile banana prawns Penaeus merguiensis from a mangrove estuary and recruitment to offshore areas in the wet-dry tropics of the Gulf of Carpentaria, Australia. Marine Ecology Progress Series, 27, 52.

Sunday JM, Bates AE, Dulvy NK (2012) Thermal tolerance and the global redistribution of animals. Nature Climate Change, 2, 686-690.

Sunday JM, Pecl GT, Frusher S et al. (2015) Species traits and climate velocity explain geographic range shifts in an ocean-warming hotspot. Ecology Letters, 18, 944-953.

Van Schaik CP, Terborgh JW, Wright SJ (1993) The phenology of tropical forests: adaptive significance and consequences for primary consumers. Annual Review of Ecology and Systematics, 24, 353-377.

Wolkovich EM, Cook B, Allen J et al. (2012) Warming experiments underpredict plant phenological responses to climate change. Nature, 485, 494-497.

\section{Supporting Information}

Additional Supporting Information may be found in the online version of this article:

Table S1. Distribution studies, rates of change and factors used in the analysis.

Table S2. Phenology studies, rates of change and factors used in the analysis. 